\title{
The Role of Imaging to Support Pulmonary Vein Isolation Procedures: Review of the Literatures
}

\section{Ahmed Mokhtar Abd El Azeem*}

Department of Cardiology and Angiology, Alexandria University Chamblion Street, el azareeta, Alexandria Governorate, Egypt.

${ }^{*}$ Corresponding author: Ahmed Mokhtar Abd El Azeem, Department of Cardiology and Angiology, Alexandria University Chamblion Street, el azareeta, Alexandria Governorate, Egypt. Tel: +20 3 4862506; E-mail: A_elmekawy00@Alexmed.edu.eg

Received: May 10, 2017; Accepted: June 28, 2017; Published: July 07, 2017

Citation: Abd El Azeem AM (2017) The Role of Imaging to Support Pulmonary Vein Isolation Procedures: Review of the Literatures. Ann Clin Lab Res. 5: 2

\section{Abstract}

Atrial fibrillation (AF) ablation is a complex procedure that requires access to the left atrium and Pulmonary Vein Isolation (PVI). The results of these procedures are highly variable due to the heterogeneous nature of these veins as well as the ablation techniques. Identification of this complex anatomy and the relationship to the surrounding structures can help to increase the success of these procedures and to reduce the risk and complications of these ablation procedures. Thus, imaging techniques play an important role in procedural planning. Preprocedural imaging is important to rule out thrombosis, assess the anatomy of the pulmonary veins (PVs) and left atrium (LA), and evaluate the extent of underlying arrhythmic substrate. Integration of imaging with ablation navigation systems can increase the efficacy and safety of catheter ablation as well as decrease fluoroscopy time. Postoperative imaging techniques are useful to detect complications. This paper briefly reviews the different imaging tools for ablation of AF.

Keywords: Atrial fibrillation ablation; Pulmonary vein isolation; Imaging; Cardiac computed tomography; Cardiac MRI, 3D Transesophageal echocardiography; Intracardiac echocardiography.

\section{Introduction}

Atrial fibrillation (AF) is a common cardiac rhythm disturbance with increasing prevalence and associated with increased morbidity and mortality. Catheter ablation of AF is indicated for patients with drug refractory highly symptomatic paroxysmal AF when done in experienced centers by trained personnel [1]. Pulmonary Vein Isolation (PVI) is considered the corner stone for AF ablation procedures [2]. However, ablation success rates remain in the range of $70 \%$ with worse outcomes in patients with persistent form of AF. Redo procedures commonly required to achieve long term success. Imaging for detailed atrial tissue characteristics, such as fibrosis, are commonly used to allow better understanding of the patient specific atrial substrate that contributes to the success and failure of AF ablation [3]. The proximity of the left atrium to surrounding structures such as esophagus emphasizes the need for better imaging techniques than fluoroscopy to minimize the complications [4]. In this paper, we will review clinical utility of various imaging techniques before and after ablation of AF.

\section{Image Role Pre, Intra and Post- Procedural}

Pre-procedural cardiac imaging enables specific information to be obtained regarding the PVs and left atrium (LA) prior to AF ablation: (1) the number of PVs and branching pattern, (2) individual PV ostial measurements, (3) measurement of LA dimensions, (4) identification of LA appendage thrombus, (5) identification of anatomic anomalies in the PVs, (6) demonstrate the anatomy of the interatrial septum which may help guide trans-septal puncture, and (7) provide anatomic knowledge of structures such as the esophagus to minimize complications including atrioesophageal fistula caused by burns to the esophagus during ablation [5].

Intraprocedural imaging can guide trans-septal puncture, guide cryoballoon placement and assess PV occlusion with the balloon, can be superimposed on electromagnetic system with subsequent reduction in fluoroscopy time and can allow early detection of procedural complications.

Post-operative imaging is commonly used to detect postprocedural complications like PV stenosis, atrioesophageal fistula, Transient Ischemic Attack (TIA) and stroke [6].

\section{Role of Echocardiography Modalities}

Prior to AF ablation all patients should undergo a transthoracic echocardiogram to evaluate significant underlying structural heart disease. Other data that could be obtained from echocardiography evaluation and can affect ablation outcome includes LA dimension, left ventricular (LV) filling pressure, LV ejection fraction, etc. $[1,4]$.

The role of LA mechanical dyssynchrony by speckle tracking was in the recurrence after AF ablation was investigated and it 
was found that AF recurrence had significant dyssynchrony compared to those in sinus rhythm [7].

In a Meta-analysis of 8 studies evaluating the role of LA strain in predicting outcomes after AF ablation, it was found that lower LA strain was associated with higher AF recurrence [8].

More recently, a study evaluating the effect of epicardial fat thickness measured by echocardiography in the end systole as a predictor of outcome after AF cryoablation reported that epicardial fat thickness predicted AF recurrence and was associated with higher $C$ reactive protein addressing the role of inflammation in AF [9].

Transesophageal echocardiography (TEE) is an important pre- and intraprocedural imaging tool for AF ablation. It is the gold standard tool to rule out the presence of Left Atrial Appendage (LAA) thrombus. It also provides anatomic data regarding the LA and PVs. TEE can be used to guide the transseptal puncture and ablation catheter navigation [6].

Advantages of TEE include its wide availability, familiarity with the technique for most institutions, and absence of radiation exposure.

\section{Real-time three-dimensional transesophageal echocardiography}

(RT3DTEE) has become available for clinical practice offering clear and detailed imaging of the cardiac anatomy. This technology permits direct visualization of the cardiac structures in a 3D perspective with better spatial relationship among structures, and more accurate and reliable measurements of cardiac structures than two-dimensional (2D) TEE.

- 3D TEE can be used preoperative to visualize PV ostia and surrounding structures. PV anomalies including common ostia and right-sided accessory veins could be identified with RT 3D TEE. Importantly, performing a 3D preprocedural analysis with the RT 3D TEE might bear the advantage of not having to perform a computed tomography (CT) scan or a magnetic resonance imaging (MRI). This can reduce time, cost and X-ray exposure in the case of CT scan imaging. However, it should be mentioned that CT or MRI imaging might be superior to RT 3D TEE in adequately defining the features of the anatomical variations.

- RT3DTEE leads to uneventful trans-septal (TS) access by clearly documenting the classical 'tenting' effect of the fossa ovalis (FO) indicating the correct TS position (Figure 1).

- Assessment of pulmonary vein occlusion with the cryoballoon can be done using the color doppler acquisition of the full volume in $3 D$, which shows exactly the anatomical location of eventual PV-LA leakage (Figure 2).

- Use of RT3DTEE can reduce the AF ablation complications through preforming safe TS puncture which is considered as a major source of procedure related complication. RT 3D
TEE can avoid inserting the balloon in the LAA as it is perfectly distinguishing LAA from the left-sided veins. Finally, like other echocardiographic techniques it can constantly monitor the pericardial cavity; therefore, potentially identifying effusion in its very early stages. On the other side, oesophageal hematoma has been described as a potential complication in TEE-guided AF ablation. Although rare, this complication can result in significant morbidity with long-term clinical manifestations including oesophageal stricture and persistent dysmotility [10].

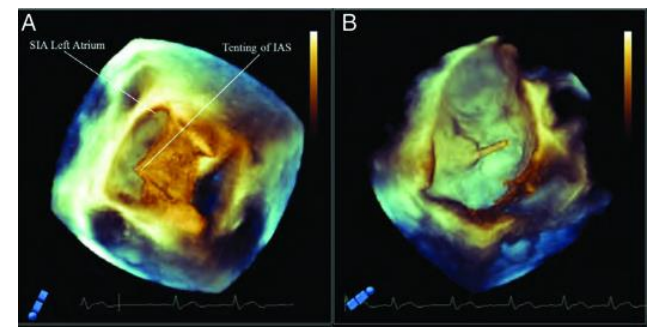

Figure 1 Performing TS puncture with real time 3D TEE. Ottaviano et al. in 2013.

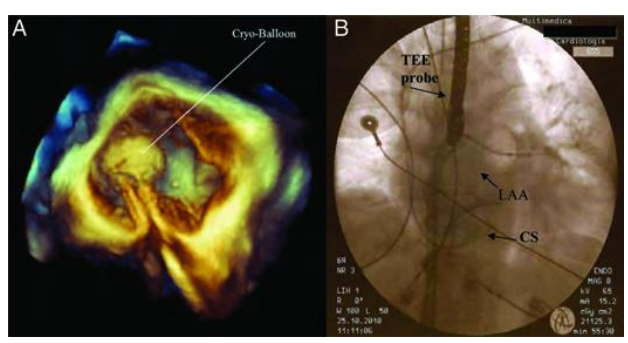

Figure 2 Perfect PV occlusion evaluated by 3D TEE and by angiography. Ottaviano et al. in 2013.

\section{Intracardiac echocardiography (ICE) / 3D- intracardiac echocardiography}

Intracardiac echocardiography is routinely used in many EP laboratories for intraprocedural imaging.

- Trans-septal puncture can be easily performed by direct ultrasound visualization of the FO, especially during needle and sheath passage, an important consideration in difficult cases such as in patients with atrial septal defect repair where a synthetic patch may present an otherwise unsolvable obstacle.

- It provides excellent anatomical details of the interatrial septum, PV antra, ridges, and other important parts of the LA allowing the operator to fine tune the ablation catheter position to achieve antral PV isolation or target carinal areas (Figure 3) [6,11].

- ICE may be used to avoid catastrophic complications such as atrioesophageal fistula by providing the location of vital surrounding structures and their relationship to the LA.

- ICE has been also used to detect pericardial effusion, thrombus formation, and to titrate the RF energy delivery 
to avoid microbubble appearance or char formation. Doppler modality could be used anytime during the procedure to check PV velocities. Experience showed that PV ostial peak velocity increasing to more than $150 \mathrm{~cm} / \mathrm{s}$ and doppler pattern of turbulent flow indicate a moderate narrowing effect. Significant stenoses are associated with higher peak flow velocities [10].

- 3D intracardiac echocardiography: Two-dimensional ICE imaging has several limitations. For example, curved catheter shaft often cannot be fully visualized with single plane image, thus necessitating acquisition of 3D imaging planes. Reliable 3D imaging of cardiac chambers may obviate the need for virtual Electroanatomic Mapping (EAM) and make any mapping or ablation procedure more precise and faster [4].

- ICE technology is improving. Innovations include better transducers with higher resolution, ultrasound probes incorporating a port for a radiofrequency wire allowing simultaneous visualization and ablation, percutaneous intrapericardial echocardiography, and 4D ICE with gated reconstruction of cardiac motion and ICE image integration with EAM. The main advantages of ICE are no need for anesthesia and full control over imaging by an electrophysiologist [6,11-13].

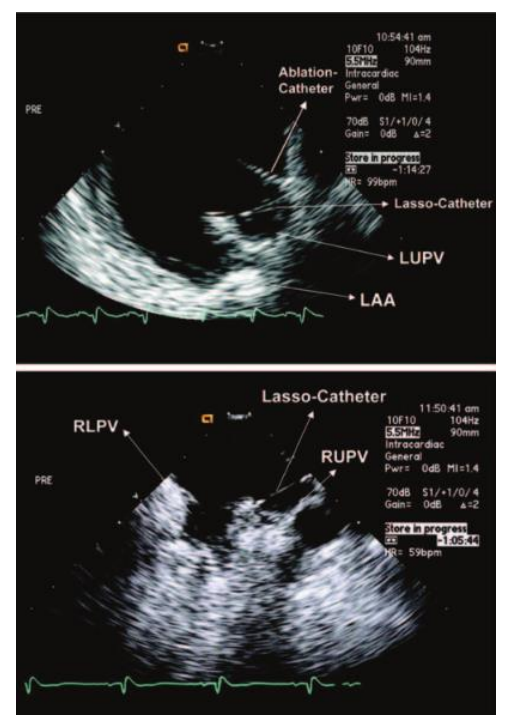

Figure 3 ICE images in the upper one ablation catheter in the left superior PV, lower one showing lasso catheter in right inferior PV. Marrouche et al. in 2003.

\section{Role of Computed Tomography (CT)}

Cardiac CT scan can aid in AF ablation through the wide range of data obtained regarding $\mathrm{PV}$ and LA anatomy, identification of relation to adjacent structures to prevent complications and allow safe navigation of ablation catheters, diagnosis of post-procedural complication.

- CT angiography (CTA) provides a detailed 3D perspective of the left atrium and the number, location, size, and geometry of the pulmonary veins. CTA appears to characterize complex left atrial anatomy better than 2D imaging modalities such as fluoroscopy and echo. The common occurrence of pulmonary vein variants can be easily and accurately identified. Supernumerary PVs most often right sided occur in $10 \%$ to $39 \%$ of people in 3D angiogram studies. AF triggering foci can be located within these additional veins requiring their isolation. Early branching from the PV ostium can also be seen and appears more common for the right PVs. Being alerted to their presence is important because ablating near small or early branching PVs increases the risk of PV stenosis. A left common PV trunk results in a broad PV junction and is present in $12 \%$ to $29 \%$ of people in $3 \mathrm{D}$ angiogram studies (Figures 4 and 5) [3].

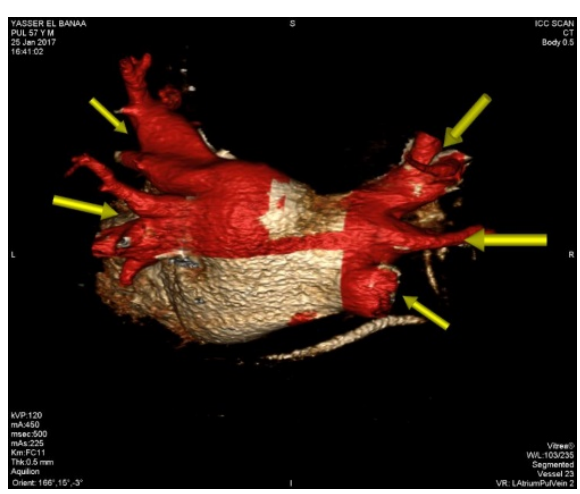

Figure 4 3D volume reconstructed cardiac CT showing supernumerary right sided PVs and early branching left inferior PV.

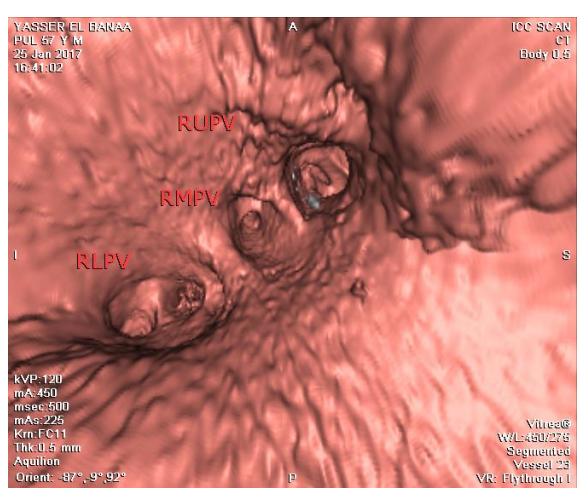

Figure 5 CT showing the ostia of 3 right sided PV.

- Sizing of the PVs provided by 3D imaging may become even more important with the advance of complex ablation tools such as cryoballoon. Therefore, pre-procedural determination of the tool size becomes more important.

- CT has demonstrated significant variations in atrial wall thickness between different regions of the left atrium, ranging from $0.5 \mathrm{~mm}$ to $3 \mathrm{~mm}$ with significant interpatient variability. Variations in regional wall thickness might be useful for directing increasing ablation power in thicker regions, such as the appendage ridge, mitral isthmus, and superior roof, to increase the likelihood of transmural 
lesion formation. Conversely, power could be decreased in thinner regions, such as the superior posterior wall, to avoid complications such as perforation [14].

- CT can also provide data concerning structural remodeling and pro-arrhythmia. Gross atrial volume and shape reflect some of aspects of pro-arrhythmic atrial substrate. For example, progressively larger atrial volume by CTA has been associated with incrementally higher odds of $A F$ recurrence following ablation. A more flattened atrial roof shape may also identify patients who require additional trigger ablation or substrate modification outside of PV isolation $[15,16]$.

- $\quad$ CT is also capable of localizing and quantifying pericardial fat, which plays a role in promoting AF. Studies by Batal et al. and Thanassoulis et al. provided evidence that the presence and quantity of pericardial fat measured by CT was associated with increasingly persistent AF forms [17-21].

- Identification of specific LA anatomy, like locations including the ridge of Marshall located between the LAA and the left PV which is not adequately appreciated on EAM, as well as the ridges separating the right middle PV with its neighboring right superior and inferior PV. These anatomical ridges are visualized well with $\mathrm{CT}$; the LAA ridge is $<5 \mathrm{~mm}$ and ridges surrounding the right middle PV superiorly and inferiorly are narrower than $2 \mathrm{~mm}$. The inability to achieve stable catheter positions at these locations may result in incomplete ablation lines and delivery of lesions within the PV, potentially resulting in PV stenosis or ablating too close to the LAA. Thus, preprocedural imaging of the LA with CT demonstrating narrow LA ridges may affect the ablation location and strategy. [5] (Figure 6).

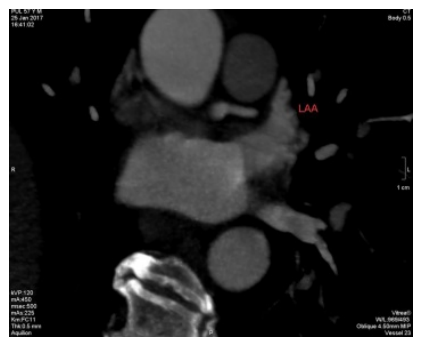

Figure $6 \mathrm{CT}$ showing the ridge between the LAA and LSPV.

- $\mathrm{CT}$ is helpful in prevention of complications. Appreciation of the relationship to surrounding anatomy is important to minimize mechanical and ablation related complications. The risk of catheter-mediated perforation can be reduced by additional localization of the PV junctions and knowledge of regional variations in atrial thickness. Ablation injury of adjacent structures can be minimized by understanding the anatomic relationships of these structures such as the course of the right phrenic nerve between the right superior PV and superior vena cava/right atrium $[3,22]$.
- $\mathrm{CT}$ is also valuable in diagnosis and prevention of pulmonary vein stenosis which can be reduced by avoiding ablation inside the PVs.

- CT can prevent oesophageal injury. Pre-procedural CT can determine the course of the esophagus and its relationship to posterior wall, upper right and upper left PVs. CT is also the diagnostic modality of choice for atrioesophageal fistula [3].

\section{Role of Cardiac Mangetic Resonanse Imaging (MRI)}

Cardiac MRI plays a crucial role in AF ablation as it can precisely delineate the anatomy of LA and PVs, evaluate atrial fibrosis which is linked to procedural success, evaluate post ablation lesion characteristics and can prevent and diagnose procedural complications.

\section{Before ablation procedure.}

- MRI angiography (MRA) provides a detailed 3D perspective of the left atrium anatomy and the number, location, size, and branching pattern of the pulmonary veins. Since an early MRA study by Kato et al. outlined PV anatomic relationships, many MRA studies have confirmed the common occurrence of pulmonary vein variants. Understanding this complex PV anatomy is mandatory for a successful PVI procedure, hence missing one of these PVs will lead to AF recurrence [23].

- MRI is useful for evaluating arrhythmogenic atrial substrate and ablation patient selection. Moreover, MRI can predict outcome after ablation. MRI can directly assess the atrial fibrotic changes that parallel the atrial fibrillation progression process. Delayed Gadolinium Enhancement MRI (DEMRI) is based on the retention of injected gadolinium contrast within myocardium that has disrupted vasculature. Oakes et al. first reported the possibility of detecting atrial fibrosis using DEMRI. They found that the extent of pre-ablation LA DEMRI enhancement was 3 to 4 times more predictive of response to drug therapy and $A F$ ablation than LA volume. In this study, post AF ablation recurrence occurred in $75 \%$ of patients with extensive enhancement, $43 \%$ of those with moderate enhancement, and only $14 \%$ of those with mild enhancement [23]. Follow-up studies by the same group have supported this finding, leading to proposal of a "fibrosis" scoring system based on total amount of DEMRI atrial enhancement (Figure 7). They reported no recurrence in patients with very low $(<5 \%)$ enhancement but $56 \%$ to $96 \%$ recurrence in those with more than $35 \%$ enhancement. [24,25]. More recently, Khurram et al. evaluated the effect of late gadolinium enhancement on AF recurrence after $\mathrm{PVI}$ and concluded that regardless of AF persistence at baseline, participants with LGE $<35 \%$ have favorable outcomes, whereas those with $L G E>35 \%$ have a higher rate of $A F$ recurrence in the first year after ablation. These findings suggest a role for: 1) patient selection for AF ablation using LGE extent; and 2) substrate modification in addition to 
pulmonary vein isolation in patients with LGE extent exceeding $35 \%$ of left atrial myocardium. These results suggest the limitation of relying on standard paroxysmal and persistent clinical categories for predicting response to AF ablation [26].

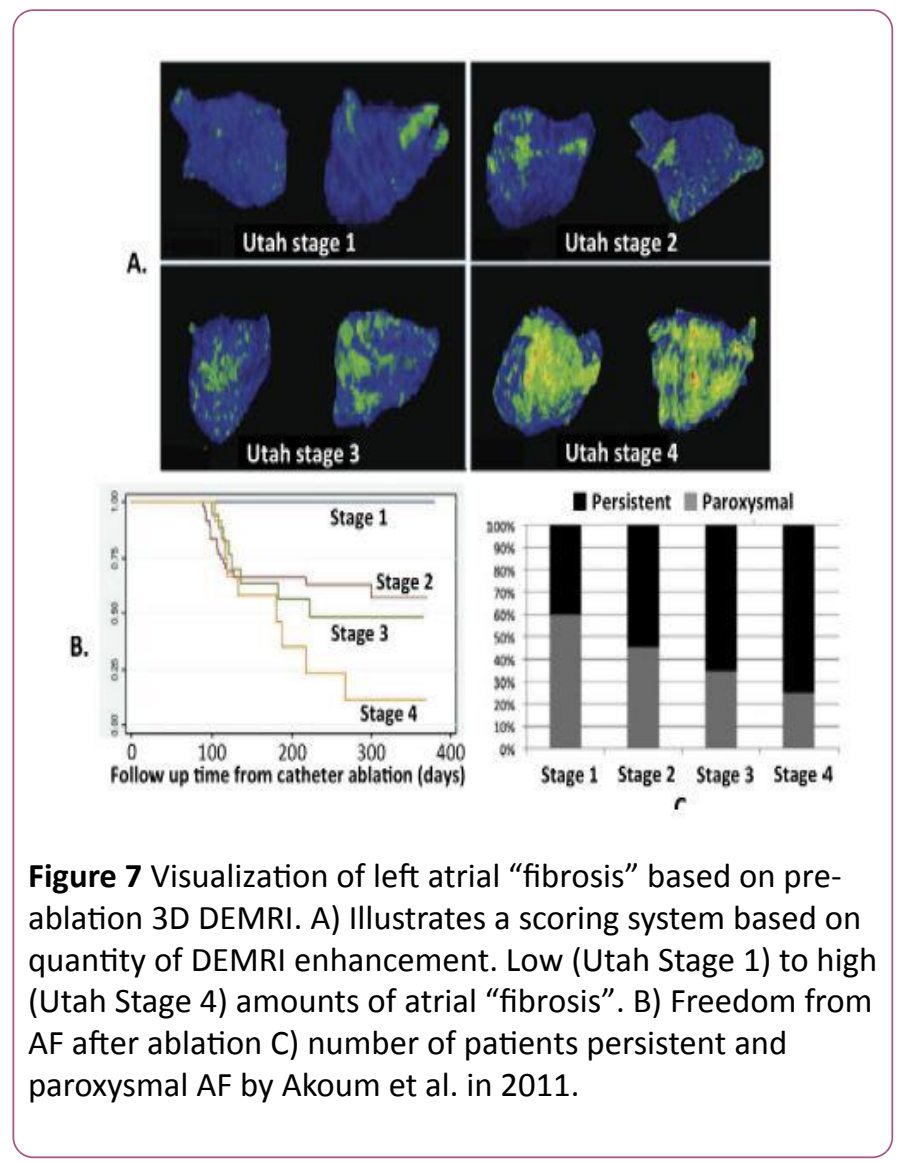

- In another study by Suksaranjit et al. they found that in AF patients without history of myocardial infarction, LV-LGE is a significant independent predictor of AF recurrence after ablation therapy [27].

- Using MRI, Wong et al. found that pericardial fat volume was associated with the presence and chronicity of AF. They also found that pericardial fat deposits were predictive of AF recurrence after ablation [28].

\section{Post procedural role of MRI.}

- MRI can be used also to evaluate the success of circumferential ablation line 1. Recovery of PV conduction is typical in patients who undergo repeat $A F$ ablation despite documentation of electrical pulmonary vein isolation after previous procedures. This is likely due to resolution of transient factors such as edema that can resolve in the days to weeks following ablation. MRI has the most established history for assessing lesion characteristics after ablation.

- In 2007 Peters et al. introduced the concept of highresolution atrial ablation lesion imaging following PVI using DEMRI. McGann et al. used a standardized workflow for 3D ablation lesion quantification and visualization. They found that despite a uniform and extensive ablation protocol, there was significant patient-to-patient variation in postablation LA enhancement. They also found the greater the amount of post-ablation enhancement, the less AF recurrence after ablation. Taclas et al. found that $20 \%$ of lesions they marked with EAM did not have evidence of associated DEMRI atrial enhancement. They also related more gaps in DEMRI enhancement to greater $A F$ recurrence after ablation. Conversely, Badger et al. found that achieving circumferential DEMRI enhancement around all pulmonary veins was difficult and only seen in $7 \%$ of patients after initial ablation. However, all patients with circumferential enhancement of all veins were free from $\mathrm{AF}$ at one year. Akoum et al. combined pre- procedure DEMRI fibrosis assessment and post procedure DEMRI lesion assessment. After PVI plus extensive substrate ablation, they found that achieving circumferential DEMRI enhancement around the PVs predicted procedural success only in patients with relatively less DEMRI atrial fibrosis. However, in patients with more atrial fibrosis only overall post-ablation atrial enhancement was predictive of success. This study suggests that imaging detailed atrial tissue characteristics may be useful for identifying patients who are likely to require an ablation strategy beyond PVI. Badger et al. reported that the amount of DEMRI enhancement seen within 24 hours of ablation was significantly greater than that seen at 3 and 6 months after ablation. This reflects the transient atrial injury that does not correlate well with eventual atrial scarring. A follow-up study by McGann et al. suggested that non-enhancing (dark areas of no reflow) regions on DEMRI might better reflect regions that will eventually form durable ablation lesions [29-34].

- MRI can also prevent ablation complications by means of better identification of the anatomy, evaluation of the proximity of the nearby structure such as oesophagus, aorta, phrenic nerve and evaluation of atrial wall thickness to prevent perforation.

- The shift towards a more extensive ablation in the LA, particularly in persistent atrial fibrillation, raises concern for the adversely impact on atrial structure and function. Using post-ablation DEMRI to quantify LA scarring after ablation, Wylie et al. found a linear correlation between more extensive ablation and reduced atrial ejection fraction (EF). Though another meta-analysis reported overall improvement in atrial function following successful ablation $[35,36]$.

\section{MRI for repeat procedures}

- In a study by Bisbal et al. evaluating the role of cardiac MRI in repeat ablation procedures, they found that DEMRI can successfully guide repeated PVI procedures by accurately identifying and localizing gaps and may reduce procedural duration and radiofrequency application time [37]. 


\section{Merge Technologies}

\section{CT and cardiac MRI fluoroscopy merge}

- The idea of overlaying a 3D anatomical shell of the heart chamber on live fluoroscopy is attractive. Both CT and MRI data can be segmented and registered to a live fluoroscopy screen. Early experience confirmed high accuracy of catheter navigation within properly registered images. A randomized comparison with EAM demonstrated similar radiation exposure when using CT overlay on live fluoroscopy as the only imaging guide and a shorter procedural time. These overlay tools are relatively inexpensive as they do not require specialized mapping catheters or patches. Disadvantages of purely $X$ ray-based methods include radiation exposure that may not be negligible with non-gated CT. Another disadvantage of CT or MRI-based system is the lag between the actual imaging and the procedure day $[6,38,39]$ (Figure 8).

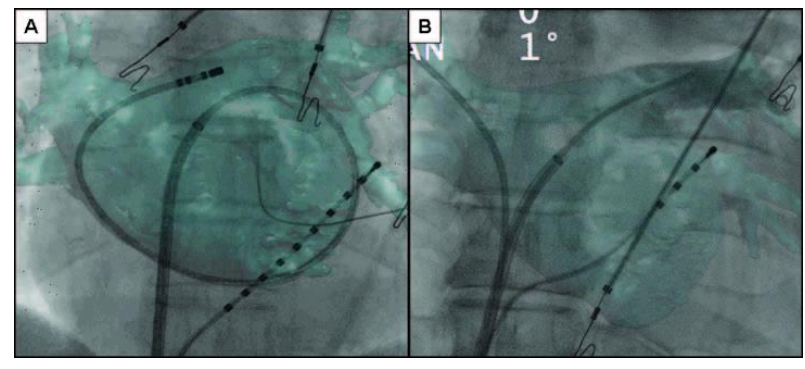

Figure 8 showing CTA merged with fluoroscopy by Knecht et al. in 2008 .

\section{CT and cardiac MRI merged with electroanatomic mapping}

- Fusion of 3D anatomical data with EAM can theoretically correct anatomical imperfections of EAM methods while providing almost real-time non-fluoroscopic intraprocedural navigation guidance. Early publications showed feasibility and high accuracy of CT/MR and EAM fusion. Minimal discrepancy (about $2 \mathrm{~mm}$ ) between the CT shell and mapping points. Accurate superimposition of these fused images is a complex issue. It requires sophisticated computer algorithms and sometimes imagination. Reconstructed 3D shells of the chamber of interest, obtained by different methods, may simply be different depending on acquisition time, volume status, respiratory movement, etc. [40-43].

\section{Real-Time CMR: The Future of the Electrophysiology Lab?}

- The purpose of the MRI-EP suite is to avoid radiation exposure. Further, accurate definition of cardiac structures and arrhythmic substrates such as scar and fibrosis, visualization of catheter position, and the possibility to monitor the efficiency of radiofrequency applications by observing the lesion formation makes this technology very attractive [44].

- Initial experiences with ablation of arrhythmias using realtime CMR guidance indicate that it seems to be feasible and safe.

- Radiation exposure has been significantly reduced with the use of electroanatomic mapping systems; however, the use of interventional CMR would completely eliminate radiation exposure. Efficacy could be improved by identifying areas of edema with incomplete isolation; advances and improvements in real-time CMR guidance might be useful to detect gaps during the index procedure despite transient electrical isolation due to edema or nontransmural lesions. This would be a very important step toward improving the long-term outcome of AF ablation.

- However, despite these potential improvements, the implementation of a CMR suite is still a challenge. Several limitations must be overcome: the need for compatible equipment (12-lead ECG, catheters, wires, and ablation systems) and improved signal filtering to allow reliable signal interpretation, as well as the guarantee of patient safety: current defibrillation systems are not safe inside the CMR [45-47].

\section{Special Considerations}

Though in practice CT and MRI are often interchangeable, these modalities have some advantages and disadvantages. CT has the advantage of short imaging times and high spatial resolution. Current CT systems can generate 3D angiograms with $0.5 \mathrm{~mm} \times 0.5 \mathrm{~mm} \times 0.5 \mathrm{~mm}$ resolution within around 10 heart beats for 64 detector systems and within one heart beat for 320 detector systems. In contrast, a 20 to 30 second breath hold is typically required for 3D MRA with a resolution around $1.5 \mathrm{~mm} \times 1.5 \mathrm{~mm} \times 1.5 \mathrm{~mm}$.

More significantly, much longer imaging times of several minutes are currently required for the "fibrosis" and ablation lesion DEMRI studies discussed above. Reliably mitigating the effects of patient and physiologic motion over these longer imaging times remains a challenge.

An advantage of MRI over CT is that it does not require ionizing radiation. $\mathrm{MRI}$ also has the advantage of more flexible soft tissue contrast compared with CT. This has led to the more rapid advances in MRI of atrial substrate and ablation lesions.

The use of both CT and MRI is limited in patients with significant renal dysfunction. The incidence and severity of renal dysfunction with iodine-based $\mathrm{CT}$ contrast agents markedly increases in patients with GFR $<30 \mathrm{ml} / \mathrm{min}$.

Gadolinium MRI contrast has been associated with a very rare but serious condition, nephrogenic systemic fibrosis (NSF). The occurrence of NSF has been limited to patients with GFR $<30 \mathrm{ml} / \mathrm{min}$ and typically dialysis dependent [48-50]. 


\section{Conclusion}

- $\mathrm{AF}$ is a progressive disease and $\mathrm{AF}$ ablation procedures are increasing significantly but with disappointing results and a lot of repeat procedures.

- Pre-procedure imaging is extremely important for ablation planning to delineate the complex and variable anatomy of the left atrium, PVs, and surrounding structures. The information from pre-procedure 3D images can be incorporated into electroanatomic mapping systems to facilitate catheter navigation and reduce fluoroscopic radiation exposure.

- Post-procedure imaging can also be useful for evaluating complications such as PV stenosis, esophageal injury, and reduced atrial function. Incorporating MRI or CT information about tissue characteristics such as regional fibrosis, pericardial fat and atrial wall thickness may provide additional information for selecting ablation strategies or for guiding ablation energy delivery.

- Ablation lesion imaging is an attracting point of research toward the goal of detecting incomplete ablation regions to guide additional lesion placement.

- Advances in real-time imaging modalities such as ICE and $\mathrm{MRI}$, alternative ablation energy sources and techniques, and better understanding of AF pathophysiology altogether are crucial to achieve a more uniformly successful treatment for atrial fibrillation.

\section{References}

1. Kirchhof $P$, Benussi S, Kotecha D, Ahlsson A, Atar D, et al. (2016) ESC Guidelines for the management of atrial fibrillation developed in collaboration with EACTS. Eur J Cardiothorac Surg 50: 2893-2962.

2. Calkins H, Kuck KH, Cappato R, Brugada J, Camm AJ, et al. (2012) 2012 HRS/EHRA/ECAS Expert consensus statement on catheter and surgical ablation of atrial fibrillation: Recommendations for patient selection, procedural techniques, patient management and follow-up, definitions, endpoints, and research trial design. Heart Rhythm 9: 632-696.

3. Kolandaivelu A (2012) Role of Cardiac Imaging (CT/MRI) Before and after $\mathrm{rf}$ catheter ablation in patients with atrial fibrillation. Atr Fibrillation 5: 54-65.

4. Kautzner J, Peichl P (2012) The role of imaging to support catheter ablation of atrial fibrillation. Cor et vasa 54: 375-385.

5. Thai W, Wai B, Truong Q (2012) Preprocedural imaging for patients with atrial fibrillation and heart failure. Curr Cardiol Rep 14: 584-592.

6. Syros G, Orlov M (2011) Advances in imaging to assist atrial fibrillation ablation. The Journal of Innovations in Cardiac Rhythm Management 2: 570-582.

7. Loghin C, Karimzadehnajar K, Ekeruo IA, Mukerji SS, Memon NB, et al. (2014) Outcome of pulmonary vein isolation ablation for paroxysmal atrial fibrillation: predictive role of left atrial mechanical dyssynchrony by speckle tracking echocardiography. J Interv Card Electr 39: 7-15.

8. Ma XX, Boldt LH, Zhang YL, Zhu MR, Hu B, et al. (2016) Clinical relevance of left atrial strain to predict recurrence of atrial fibrillation after catheter ablation: A meta-analysis. Echocardiography 33: 724-733.

9. Canpolat U, Aytemir K, Yorgun H, Asil, Dural M, et al. (2016) The impact of echocardiographic epicardial fat thickness on outcomes of cryoballoon-based atrial fibrillation ablation. Echocardiography 33: 821-829.

10. Ottaviano L, Chierchia GB, Bregasi A, Bruno N, Antonelli A, et al. (2013) Cryoballoon ablation for atrial fibrillation guided by realtime three-dimensional transoesophageal echocardiography: A feasibility study. Europace 15: 944-950.

11. Dello Russo A, Fassini G, Casella M, Di Monaco A, Riva S, et al. (2015) Usefulness of intracardiac echocardiography during pulmonary vein isolation with the novel multipolar irrigated ablation catheter (nMARQ $\left.{ }^{\mathrm{TM}}\right)$. J Interv Card Electrophysiol 44: 39-45.

12. Marrouche NF, Martin DO, Wazni O, Gillinov AM, Klein A, et al. (2003) Phased-array intracardiac echocardiography monitoring during pulmonary vein isolation in patients with atrial fibrillation: impact on outcome and complications. Circulation 107: $2710-2716$

13. Hijazi ZM, Shivkumar K, Sahn DJ (2009) Intracardiac echocardiography during interventional and electrophysiological cardiac catheterization. Circulation 119: 587-596.

14. Beinart R, Abbara S, Blum A, Ferencik M, Heist K, et al. (2011) Left atrial wall thickness variability measured by CT scans in patients undergoing pulmonary vein isolation. J Cardiovasc Electrophysiol 22: 1232-1236.

15. Hof I, Chilukuri K, Arbab-Zadeh A, Scherr D, Dalal D, et al. (2009) Does left atrial volume and pulmonary venous anatomy predict the outcome of catheter ablation of atrial fibrillation? J Cardiovasc Electrophysiol 20: 1005-1010.

16. Burstein B, Nattel S (2008) Atrial fibrosis: Mechanisms and clinical relevance in atrial fibrillation. J Am Coll Cardiol 51: 802-809.

17. Batal O, Schoenhagen $P$, Shao M, Ayyad AE, Van Wagoner DR, et al. (2010) Left atrial epicardial adiposity and atrial fibrillation. Circ Arrhythm Electrophysiol 3: 230-236.

18. Thanassoulis G, Massaro JM, O'Donnell CJ, Hoffmann U, Levy D, et al. (2010) Pericardial fat is associated with prevalent atrial fibrillation: The Framingham Heart Study. Circ Arrhythm Electrophysiol 3: 345-350.

19. Dong J, Dickfeld T, Dalal D, Cheema A, Vasamreddy CR, et al. (2006) Initial experience in the use of integrated electroanatomic mapping with three-dimensional MR/CT images to guide catheter ablation of atrial fibrillation. J Cardiovasc Electrophysiol 17: 459-466.

20. Martinez MW, Kirsch J, Williamson EE, Syed IS, Feng D, et al. (2009) Utility of non-gated multidetector computed tomography for detection of left atrial thrombus in patients undergoing catheter ablation of atrial fibrillation. JACC Cardiovasc Imaging 2: 69-76.

21. Garcia MJ (2009) Detection of left atrial appendage thrombus by cardiac computed tomography: A word of caution. JACC Cardiovasc Imaging 2: 77-79.

22. Canpolat $U$, Aytemir $\mathrm{K}$, Hızal $M$, Hazırolan $\mathrm{T}$, Yorgun $\mathrm{H}$, et al. (2014) Imaging before cryoablation of atrial fibrillation: Is phrenic nerve palsy predictable? Europace 16: 505-510

23. Oakes RS, Badger TJ, Kholmovski EG, Akoum N, Burgon NS, et al. (2009) Detection and quantification of left atrial structural 
remodeling with delayed-enhancement magnetic resonance imaging in patients with atrial fibrillation. Circulation 119: 1758-1767

24. Mahnkopf C, Badger TJ, Burgon NS, Daccarett M, Haslam TS, et al. (2010) Evaluation of the left atrial substrate in patients with lone atrial fibrillation using delayed enhanced MRI: implications for disease progression and response to catheter ablation. Heart Rhythm 7: 1475-1481.

25. Akoum N, Daccarett M, McGann C, Segerson N, Vergara G, et al. (2011) Atrial fibrosis helps select the appropriate patient and strategy in catheter ablation of atrial fibrillation: A DE-MRI guided approach. J CardiovascElectrophysiol 22: 16-22.

26. Khurram IM, Habibi M, Gucuk Ipek E, Chrispin J, Yang E, et al. (2016) Left atrial lge and arrhythmia recurrence following pulmonary vein isolation for paroxysmal and persistent af. JACC. Cardiovascular Imaging 9: 142-148.

27. Suksaranjit P, Akoum N, Kholmovski EG, Stoddard GJ, Chang L, et al. (2015) Incidental LV LGE on CMR imaging in atrial fibrillation predicts recurrence after ablation therapy. J Am Coll Cardiol Img 8: 793-800.

28. Wong CX, Abed HS, Molaee P, Nelson AJ, Brooks AG, et al. (2011) Pericardial fat is associated with atrial fibrillation severity and ablation outcome. J Am Coll Cardiol 57: 1745-1751.

29. Peters C, Wylie JV, Hauser TH, Kissinger KV, Botnar RM, et al. (2007) Detection of pulmonary vein and left atrial scar after catheter ablation with three-dimensional navigator-gated delayed enhancement MR imaging: linitial experience. Radiology 243: 690-695.

30. McGann CJ, Kholmovski EG, Oakes RS, Blauer JJ, Daccarett M, et al. (2008) New magnetic resonance imaging-based method for defining the extent of left atrial wall injury after the ablation of atrial fibrillation. J Am Coll Cardiol 52: 1263-1271.

31. Taclas JE, Nezafat R, Wylie JV, Josephson ME, Hsing J, et al. (2010) Relationship between intended sites of RF ablation and post-procedural scar in AF patients, using late gadolinium enhancement cardiovascular magnetic resonance. Heart Rhythm 7: 489-496

32. Badger TJ, Daccarett M, Akoum NW, Adjei-Poku YA, Burgon NS, et al. (2010) Evaluation of left atrial lesions after initial and repeat atrial fibrillation ablation: lessons learned from delayedenhancement MRI in repeat ablation procedures. Circ Arrhythm Electrophysiol 3: 249-259.

33. Badger TJ, Oakes RS, Daccarett M, Burgon NS, Akoum N, et al. (2009) Temporal left atrial lesion formation after ablation of atrial fibrillation. Heart Rhythm 6: 161-168.

34. McGann C, Kholmovski E, Blauer J, Vijayakumar S, Haslam T, et al. (2011) Dark regions of no-reflow on late gadolinium enhancement magnetic resonance imaging result in scar formation after atrial fibrillation ablation. J Am Coll Cardiol 58: 177-185.

35. Wylie JV, Peters DC, Essebag V, Manning WJ, Josephson ME, et al. (2008) Left atrial function and scar after catheter ablation of atrial fibrillation. Heart Rhythm 5: 656-662.

36. Jeevanantham V, Ntim W, Navaneethan SD, Shah S, Johnson AC, et al. (2010) Meta-analysis of the effect of radiofrequency catheter ablation on left atrial size, volumes and function in patients with atrial fibrillation. Am J Cardiol 105: 1317-1326.
37. Bisbal F, Guiu E, Grand PC, Berruezo A2, Prat-Gonzalez S, et al. (2014) CMR-Guided Approach to Localize and Ablate Gaps in Repeat AF Ablation Procedure. J Am Coll Cardiol Img 7: 653-663.

38. Ector J, De Buck S, Adams J, Dymarkowski S, Bogaert J, et al. (2005) Cardiac three-dimensional magnetic resonance imaging and fluoroscopy merging: A new approach for electroanatomic mapping to assist catheter ablation. Circulation 112: 3769-3776.

39. Stevenhagen J, Van Der Voort PH, Dekker LR, Bullens RW, Van Den Bosch H, et al. (2010) Three-dimensional CT overlay in comparison to CartoMerge for pulmonary vein antrum isolation. J Cardiovasc Electrophysiol 21: 634-639.

40. Tops LF, Bax JJ, Zeppenfeld K, Jongbloed MR, Lamb HJ et al. (2005) Fusion of multislice computed tomography imaging with three-dimensional electroanatomic mapping to guide radiofrequency catheter ablation procedures. Heart Rhythm 2: 1076-1081.

41. Heist EK, Chevalier J, Holmvang G, Singh JP, Ellinor PT, et al. (2006) Factors affecting error in integration of electroanatomic mapping with CT and MR imaging during catheter ablation of atrial fibrillation. J Interv Card Electrophysiol 17: 21-17.

42. Kistler PM, Rajappan K, Jahngir M, Earley MJ, Harris S, et al. (2006) The impact of CT image integration into an electroanatomic mapping system on clinical outcomes of catheter ablation of atrial fibrillation. J Cardiovasc Electrophysiol 17: 1093-1101.

43. Dong J, Calkins H, Solomon SB, Lai S, Dalal D, et al. (2006) Integrated electroanatomic mapping with three-dimensional computed tomographic images for real-time guided ablations. Circulation 113: 186-194.

44. Grand PC, Bisbal F (2015) Current role and future prospects of magnetic resonance imaging in the field of atrial fibrillation ablation. J Atr Fibrillation. 8: 45-50.

45. Nazarian S, Kolandaivelu A, Zviman MM, Meininger GR, Kato R, et al. (2008) Feasibility of real-time magnetic resonance imaging for catheter guidance in electrophysiology studies. Circulation 118: 223-229.

46. Sommer P, Grothoff M, Eitel C, Gaspar T, Piorkowski C, et al. (2013) Feasibility of real-time magnetic resonance imagingguided electrophysiology studies in humans. Europace 15: 101-108.

47. Bhagirath $P$, Van der Graaf $M$, Karim R, Rhode K, Piorkowski $C$, et al. (2015) Interventional cardiac magnetic resonance imaging in electrophysiology. Circ Arrhythm Electrophysiol 8: 203-211.

48. Niendorf T, Sodickson DK (2008) Highly accelerated cardiovascular MR imaging using many channel technology: Concepts and clinical applications. Eur Radiol 18: 87-102.

49. Akcakaya M, Hu P, Chuang ML, Hauser TH, Ngo LH, et al. (2011) Accelerated noncontrast-enhanced pulmonary vein MRA with distributed compressed sensing. J Magn Reson Imaging 33: 1248-1255.

50. Adluru G, Chen L, Kim SE, Burgon N, Kholmovski EG, et al. (2011) Three-dimensional late gadolinium enhancement imaging of the left atrium with a hybrid radial acquisition and compressed sensing. J Magn Reson Imaging 34: 1465-1471. 